\title{
THE CONVERSE TO A THEOREM OF SHARP ON GORENSTEIN MODULES
}

\author{
IDUN REITEN ${ }^{1}$
}

\begin{abstract}
Let $A$ be a commutative local Noetherian ring with identity of Krull dimension $n, m$ its maximal ideal. Sharp has proved that if $A$ is Cohen-Macauley and a homomorphic image of a Gorenstein local ring, then $A$ has a Gorenstein module $M$ with $\operatorname{dim}_{A / m} \operatorname{Ext}^{n}(A / m, M)=1$. The aim of this note is to prove the converse to this theorem.
\end{abstract}

Throughout this note $A$ will denote a commutative local Noetherian ring with identity; $m$ will denote its maximal ideal. The concept of a Gorenstein module was introduced by Sharp in [8].

(1) Definition. A nonzero finitely generated $A$-module $M$ is called Gorenstein if the Cousin complex [7] provides a minimal injective resolution of $M$.

Sharp obtained various characterizations and properties of Gorenstein modules in [8]. In particular, he showed that for there to exist a Gorenstein $A$-module it is necessary that $A$ be Cohen-Macaulay [8, (3.9)], and he showed that a Gorenstein $A$-module has zero annihilator [8, (4.12)]. It follows that (in the notation of [8]) if $M$ is a Gorenstein module, then $\mu^{i}(m, M)=0$ if and only if $i \neq K-\operatorname{dim} A[8,(3.11)]$. We define the rank of the Gorenstein $A$-module $M$ to be $\mu^{n}(m, M)$, where $n=K$-dim $A$. In [9] Sharp proved the following

(2) Theorem. If $A$ is Cohen-Macaulay and a quotient of a Gorenstein local ring, then $A$ has a Gorenstein module of rank 1 [9, (3.1)].

The aim of this note is to prove the following converse:

(3) THEOREM. If $A$ has a Gorenstein module $M$ of rank 1, then $A$ is a quotient of a Gorenstein local ring.

Received by the editors May 27, 1971.

AMS 1970 subject classifications. Primary 13C10, 13C15, 13E05, $13 \mathrm{H} 10$.

Key words and phrases. Commutative Noetherian ring, Cohen-Macaulay ring, Gorenstein ring, Gorenstein module.

${ }^{1}$ Research supported by NSF grant GP-21335. The author is grateful to R. Y. Sharp, whose helpful remarks and suggestions resulted in an improvement in the presentation of this paper.

(c) American Mathematical Society 1972 
The notation will be the same as that in [8], with the following exception: if $M$ is a nonzero finitely generated $A$-module, the notation $\operatorname{depth}_{A} M$ will be used instead of $\operatorname{codh}_{A} M$.

(4) The principle of idealization, introduced by Nagata (see [6, p. 2]) will be our tool. From the ring $A$ and an $A$-module $N$, we obtain a structure of a commutative ring with identity on the Cartesian product set $A \times N$. Addition is "componentwise", and multiplication is given by $\left(a_{1}, n_{1}\right) \cdot\left(a_{2}, n_{2}\right)=\left(a_{1} a_{2}, a_{1} n_{2}+n_{1} a_{2}\right) . A$ is then a quotient ring of $A \times N$, for $A \times N / 0 \times N \cong A$. Since $0 \times N$ is nilpotent, all prime ideals in $A \times N$ are of the form $\mathfrak{p} \times N$ for some prime ideal $\mathfrak{p}$ of $A$. Hence $A \times N$ is also local, and $K-\operatorname{dim} A \times N=K-\operatorname{dim} A$. Using Cohen's theorem [6, (3.4)], we easily see that $A \times N$ is Noetherian if and only if $N$ is finitely generated.

The following proposition can be deduced from [5, (10)]; however, for the sake of completeness we include a direct proof.

(5) Proposition. Suppose the A-module $N$ is nonzero. Then $A \times N$ is self-injective $\Leftrightarrow A$ is complete and $N \cong E(A / m)$ (i.e. the injective envelope of the residue field of $A$ ).

Proof. If $A$ is complete and $N=E(A / m)$, then by [2, p. 30], $T=$ $\mathrm{Hom}_{A}(A \times N, N)$ is an injective $A \times N$-module, where $A \times N$ is regarded as an $A$-module by means of the natural ring homomorphism $A \rightarrow A \times N$. Now, by $[4,(3.7)]$, the natural $A$-homomorphism $A \rightarrow \operatorname{Hom}_{A}(N, N)$ is an isomorphism. Consequently, there result $A$-module isomorphisms $T \stackrel{\sim}{\rightarrow}$ $N \oplus \operatorname{Hom}_{A}(N, N) \sim N \rightarrow A$, and a straightforward computation shows that the resulting isomorphism $T \rightarrow A \times N$ is actually an $A \times N$-isomorphism.

Conversely, if $A \times N$ is self-injective, then again by [2, p. 30], since $A \times N / 0 \times N \cong A, \operatorname{Hom}_{A \times N}(A \times N / 0 \times N, A \times N) \cong \operatorname{Ann}_{A} N \times N$ is an injective $A$-module. Since $A$ is local and $N \neq 0, A_{A n} N=0$. Furthermore the natural homomorphism $A \rightarrow \operatorname{Hom}_{A}(N, N)$ is surjective. For let $f: N \rightarrow N$ be an $A$-homomorphism. The mapping $g: 0 \times N \rightarrow A \times N$ given by $(0, n) \rightarrow$ $(0, f(n))$ is an $A \times N$-homomorphism; hence, since $A \times N$ is self-injective, $g$ can be extended to an $A \times N$-homomorphism $g^{\prime}: A \times N \rightarrow A \times N$. It follows that $f$ is just multiplication by some element $a \in A$. Hence, since $\operatorname{Ann}_{A} N=$ (0), $N$ is an injective $A$-module for which the natural homomorphism $A \rightarrow \operatorname{Hom}_{A}(N, N)$ is an isomorphism. Using [4, (3.7)] and the now established fact that the endomorphism ring of $N$ is local, we conclude that $N \cong E(A / m)$ and $A$ is complete.

(6) Corollary. Suppose $A$ is an Artin local ring, $N \neq(0)$ an A-module. $A \times N$ is self-injective $\Leftrightarrow N \cong E(A / m)$.

We remark that a direct proof of the fact that if $A$ is an Artin local ring, then $A \times E(A / m)$ is self-injective appears in [3, p. 14]. 
(7) TheOrem. Suppose $A$ is a Cohen-Macaulay ring, having Krull dimension $n$, and $M$ a nonzero finitely generated A-module. Then $A \times M$ is Gorenstein $\Leftrightarrow M$ is a Gorenstein module of rank 1 .

Proof. Assume first that $M$ is a Gorenstein module of rank 1 . Then by $[8,(3.11)]$, depth $_{A} M=$ depth $A=n$; hence we can find $\left(a_{1}, \cdots, a_{n}\right)$ an $A$-sequence and $M$-sequence (see [8, (1.7)]). Then an easy computation shows that $\left(a_{1}, 0\right), \cdots,\left(a_{n}, 0\right)$ is an $A \times M$-sequence, and

$$
\begin{aligned}
& A \times M /\left(\left(a_{1}, 0\right), \cdots,\left(a_{n}, 0\right)\right) \\
& \quad \cong A /\left(a_{1}, \cdots, a_{n}\right) \times M /\left(a_{1}, \ldots, a_{n}\right) M=A^{\prime} \times M^{\prime}
\end{aligned}
$$

Since $M$ is Gorenstein of rank 1 , and $\mu_{A}^{n+i}(m, M)=\mu_{A^{\prime}}^{i}\left(m^{\prime}, M^{\prime}\right)$ for all $i \geqq 0$ (see $[1,(2.6)]$ ), we find that $M^{\prime}$ is a Gorenstein $A^{\prime}$-module of rank 1 . Hence $M^{\prime} \cong E\left(A^{\prime} / m^{\prime}\right)$, since $K-\operatorname{dim} A^{\prime}=0$ (see [8, (3.11)]). Now $A^{\prime} \times M^{\prime}$ is self-injective by (6), hence, again using $[1,(2.6)], A \times M$ is a Gorenstein ring.

Now assume conversely that $A \times M$ is Gorenstein. Let $k=\operatorname{depth}_{A} M \leqq n$. Let $\left(a_{1}, \cdots, a_{k}\right)$ be an $A$-sequence and $M$-sequence, and as before consider $A \times M /\left(\left(a_{1}, 0\right), \cdots,\left(a_{k}, 0\right)\right)=A^{\prime} \times M^{\prime}$. If depth $\left(A^{\prime} \times M^{\prime}\right)=n-k>0$, choose an element $\left(a^{\prime}, m^{\prime}\right)$ which is $A^{\prime} \times M^{\prime}$-regular. Then it is easily seen that $a^{\prime}$ must be $M^{\prime}$-regular, a contradiction to the fact that depth ${ }_{A} M=k$. Hence depth $\left(A^{\prime} \times M^{\prime}\right)=0$, so that $n=k$; and $A^{\prime} \times M^{\prime}$ is self-injective. Then (6) implies that $M^{\prime}=E\left(A^{\prime} / m^{\prime}\right)$; hence $M$ is a Gorenstein module of rank 1 .

(8) Corollary. ${ }^{2}$ If $A$ has a Gorenstein module $M$ of rank 1 , then $A$ is a quotient of a Gorenstein local ring.

Sharp has informed me that he has obtained the following extension of (2) for a commutative Noetherian ring $B$ : If $B$ is Cohen-Macaulay and is a quotient of a Gorenstein ring, then $B$ has a Gorenstein module $M$ for which $\mu^{h t \mathfrak{p}}(\mathfrak{p}, \Omega)=1$ for all $\mathfrak{p} \in \operatorname{Spec} B$. The converse of this result can be obtained from (7) by straightforward use of localization. Combining the results of these investigations, we obtain the following

(9) Corollary. Suppose $B$ is a commutative Noetherian ring. Then there exists a Gorenstein B-module $M$ having the property that $\mu^{\text {htp }}(\mathfrak{p}, M)=1$ for all $\mathfrak{p} \in \mathrm{Spec} B$ if and only if $B$ is a Cohen-Macaulay ring which can be expressed as a homomorphic image of a Gorenstein (commutative Noetherian) ring.

\footnotetext{
${ }^{2}$ This result has been obtained independently by H. B. Foxby of Copenhagen.
} 


\section{REFERENCES}

1. H. Bass, On the ubiquity of Gorenstein rings, Math. Z. 82 (1963), 8-28. MR 27 \#3669.

2. H. Cartan and S. Eilenberg, Homological algebra, Princeton Univ. Press, Princeton, N.J., 1956. MR 17, 1040.

3. T. H. Gulliksen, Massey operations and the Poincaré series of certain local rings, Preprint Series, No. 7, University of Oslo, Oslo, Norway, August 1970.

4. E. Matlis, Injective modules over Noetherian rings, Pacific J. Math. 8 (1958), 511-528. MR 20 \#5800.

5. B. J. Müller, On Morita duality, Canad. J. Math. 21 (1969), 1338-1347.

6. M. Nagata, Local rings, Interscience Tracts in Pure and Appl. Math., no. 13, Interscience, New York, 1962. MR 27 \#5790.

7. R. Y. Sharp, The Cousin complex for a module over a commutative Noetherian ring, Math. Z. 112 (1969), 340-356.

8. ——, Gorenstein modules, Math. Z. 115 (1970), 117-139.

9. - On Gorenstein modules over a complete Cohen-Macaulay ring, Oxford Quart. J. Math. Oxford Ser. (2) 22 (1971).

Department of Mathematics, University of Illinois, Urbana, Illinois 61801

Current address: Department of Mathematics, Brandeis University, Waltham, Massachusetts 02154 